\title{
Seven Microaneurysms: Description of an Experimental Rodent Model for Neurovascular Training
}

\author{
Pablo GARCÍA FEIJOO ${ }^{1}$, Fernando Eliseo CARCELLER BENITO ${ }^{1}$, Neeraj KALRA ${ }^{2}$, \\ Germán PÁUCAR CÁRDENAS ${ }^{3}$, Miguel SÁEZ ALEGRE ${ }^{1}$, Catalina VIVANCOS SÁNCHEZ ${ }^{1}$, \\ Víctor RODRÍGUEZ DOMÍNGUEZ¹, José María RODA FRADE¹
}

${ }^{1}$ La Paz University Hospital, Department of Neurosurgery, Madrid, Spain

${ }^{2}$ Leeds General Infirmary, Department of Neurosurgery, Leeds, UK

${ }^{3}$ Hospital Nacional Alberto Sabogal Sologuren, Department of Neurosurgery, Lima, Peru

This study has been presented as an e-poster in XXIII SENEC Congress on 2019 at Salamanca, Spain.

Corresponding author: Pablo GARCÍA FEIJOO g.feijoo.neurosurgery@gmail.com

\section{ABSTRACT}

AIM: To demonstrate the microsurgical procedures, and to evaluate the feasibility of living models of experimental neurovascular training by developing new complex vascular exercises mimicking the most common intracranial aneurysms.

MATERIAL and METHODS: The procedures were performed under a Zeiss (OPMI pico f170) microscope using basic microsurgery instruments, 10/0 Nylon and blue Polypropylene micro-sutures. We selected adult albino Wistar rats weighing between 258 and $471 \mathrm{~g}$ each. Seven different aneurysm types were created using carotid, jugular, cava, aorta and femoral vessels.

RESULTS: Seven types of aneurysm were designed and created in the rat with a high-medium successful rate. There are differences in terms of realism and the difficulty of performance, according to the different types: lateral wall, bifurcation, top of the basilar, fusiform, fusiform + involved branch, Anterior Communicating Artery (ACOA) and giant. The steps and technical issues to produce these exercises are described.

CONCLUSION: We show the feasibility of creating several types of aneurysm using different vessels in a rodent model. Training on these models help to improve microsurgical skills, allowing safe practice for neurosurgeons in all stages of their career.

KEYWORDS: Microsurgery, Aneurysm, Rat, Anastomosis, Neurovascular

ABBREVIATION: ACoA: Anterior Communicating Artery

\section{INTRODUCTION}

$\mathrm{O}$ ne of the most accepted ways to train on microsurgery is in the laboratory by performing experimental vascular exercises.

Microvascular suturing and by-pass procedures are distinctive from the rest of neurosurgery, where, unlike other general procedures, the surgeon often creates something that did not exist before (7), requiring a degree of imagination and creativity. Laboratory training on anastomoses can help create higher quality anastomoses. Intraoperative time is also reduced whilst maintaining good surgical outcomes by training on simulation models, which is imperative for real world operating.

\begin{tabular}{|c|c|c|c|}
\hline Pablo GARCÍA FEIJOO & (D) : $0000-0002-9052-2421$ & Miguel SÁEZ ALEGRE & (D) : 0000-0002-3903-2726 \\
\hline Fernando Eliseo CARCELLER BENITC & (1) : 0000-0002-9072-9158 & Catalina VIVANCOS SÁNCHEZ & (1) : 0000-0002-6230-9287 \\
\hline Neeraj KALRA & (D) : 0000-0002-6737-6219 & Víctor RODRÍGUEZ DOMÍNGUEZ & (1) : 0000-0002-1688-044X \\
\hline Germán PÁUCAR CÁRDENAS & (D) : 0000-0003-3221-2078 & José María RODA FRADE & (D) : 0000-0001-9621-6129 \\
\hline
\end{tabular}


The importance of animal model training is now more important than ever due to the decline in the number of microsurgical clippings of cerebral aneurysms (6). By suturing, dissecting, sectioning, and using microsurgery tools, surgeons improve their skills when they are in the operating room. They feel more confident with the operation, their tissue handling is improved and operating under the microscope is more familiar.

When we invest time in practice on the rodent vessel, we are not only training our vascular aptitudes but also we improve the rest of the basic skills under the microscope, which is useful in several fields of neurosurgery.

The three main exercises [end-to-end, end-to-side, and sideto-side anastomoses (7)] are essential for understanding the basis of more complex procedures in microvascular surgery, however, when considered separately they are a small number of exercises in the trainee agenda.

Our aim is to describe seven aneurysm models that can provide guidance for those that are looking for more complex and challenging microsurgical training. The proposed exercises include the basic types of aforementioned anastomoses and different combinations of them using different rodent vessels for vascular recreations that mimic intracranial aneurysms.

\section{MATERIAL and METHODS}

We used adult albino Wistar rats (Rattus Norvegicus) weighing between 258 and $471 \mathrm{~g}$ each, anesthetized by using intraperitoneal Uretane. The level of anesthesia was routinely checked every 15 minutes and the vital signs (pulse, respiratory and heart rate) monitored by the same trained surgeon.
All the procedures were performed under the European directive EU 63/2010 and were approved by an Animal Ethics Committee under PROEX 160/17 according to RD 53/2013 (Spain).

The procedures were performed under a Zeiss (OPMI pico f170) microscope by using a basic microsurgical kit, 10/0 Nylon and blue Polypropylene micro-sutures.

Seven different aneurysm types were created using carotid, jugular, cava, aorta and femoral vessels. All the procedures were performed by the same trainee neurosurgeon, and supervised by a senior experienced neurosurgeon and a veterinarian.

An anterior approach to the vessels through midline longitudinal incisions was selected with the rats placed in a supine position (8).

The basic microsurgery instrument kit included: Curved microneedle holder, one curved and two straight microforceps, and a straight or curved microscissor (8).

\section{RESULTS}

Seven microaneurysms were designed and created in the laboratory, based on the types most commonly found in humans. For academic reasons we choose these seven aneurysms; among them, some may seem similar; however, they contained subtle differences in conception and technical execution.

Table I contains a brief summary of these exercises including further details we assigned based on our experience with each type.

Table I: Summary of the Characteristics of the Seven Aneurysms

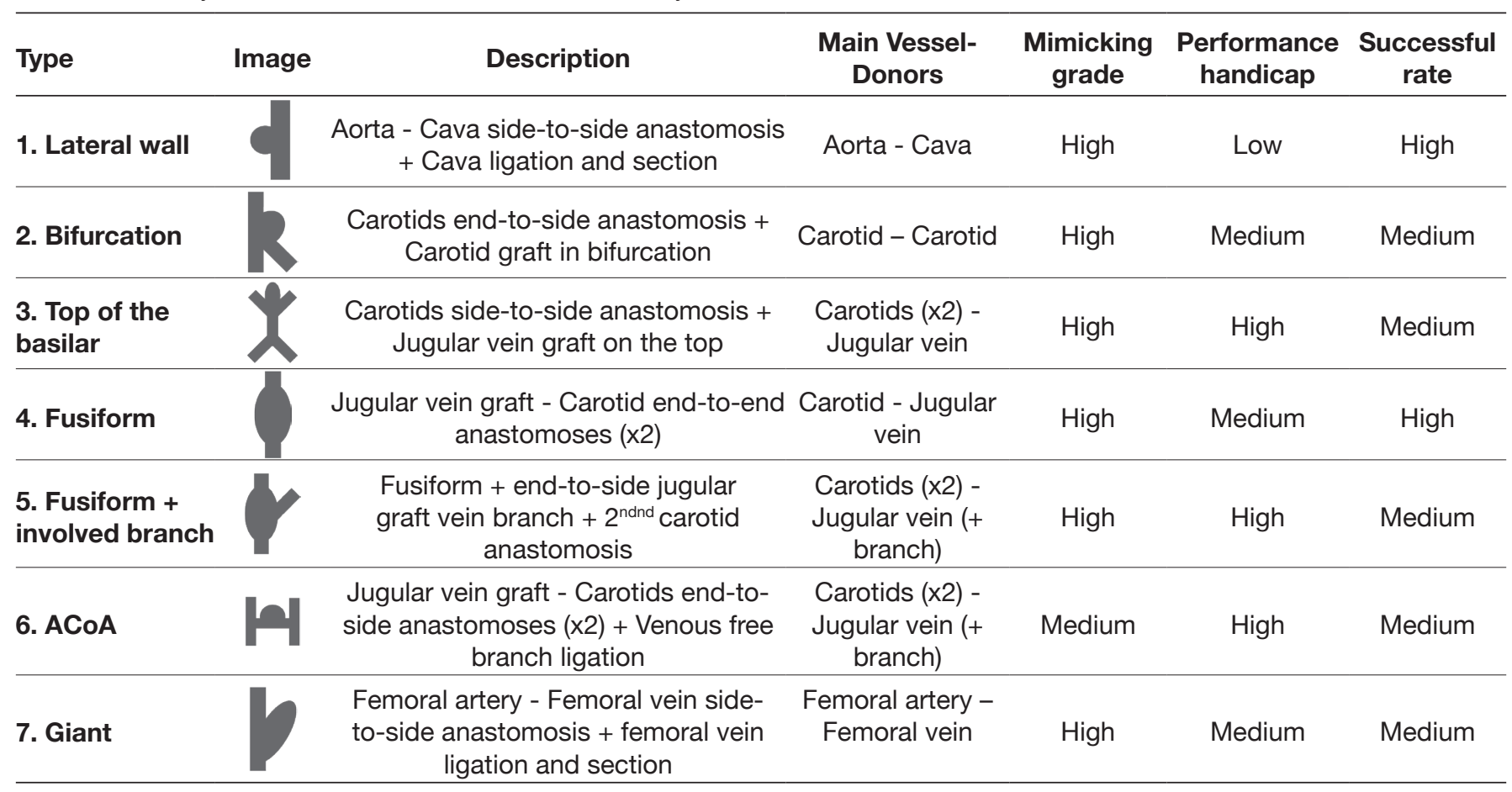

ACoA: Anterior communicating artery. 
The technical steps, numbered from 1 to 5 , and tips to perform these training exercise are described below. Visual guidance, including original images of the aneurysms is provided in Figure 1.

\section{Lateral Wall:}

Saccular aneurysms are the most common type seen in neurosurgical practice (2). As a result of its low performance difficulty, it is recommended to start with this exercise first.

1. The intra-abdominal segment of the aorta and cava is widely exposed. It is better to work in the infrarenal segment, because there are less arterial branches to be ligated.
2. After dissection and temporary clipping of both vessels, an excision arteriotomy on the medial wall of the cava and the aorta is performed.

3. A side-to-side anastomosis is performed between the aorta and cava.

4. Ligation and section of the cava above and below the anastomosis.

5. After release the temporal clipping the blood flows into the aneurysm.

This sort of exercise can be also practiced in cervical vessels.

1. A medium size jugular vein graft must be harvested.

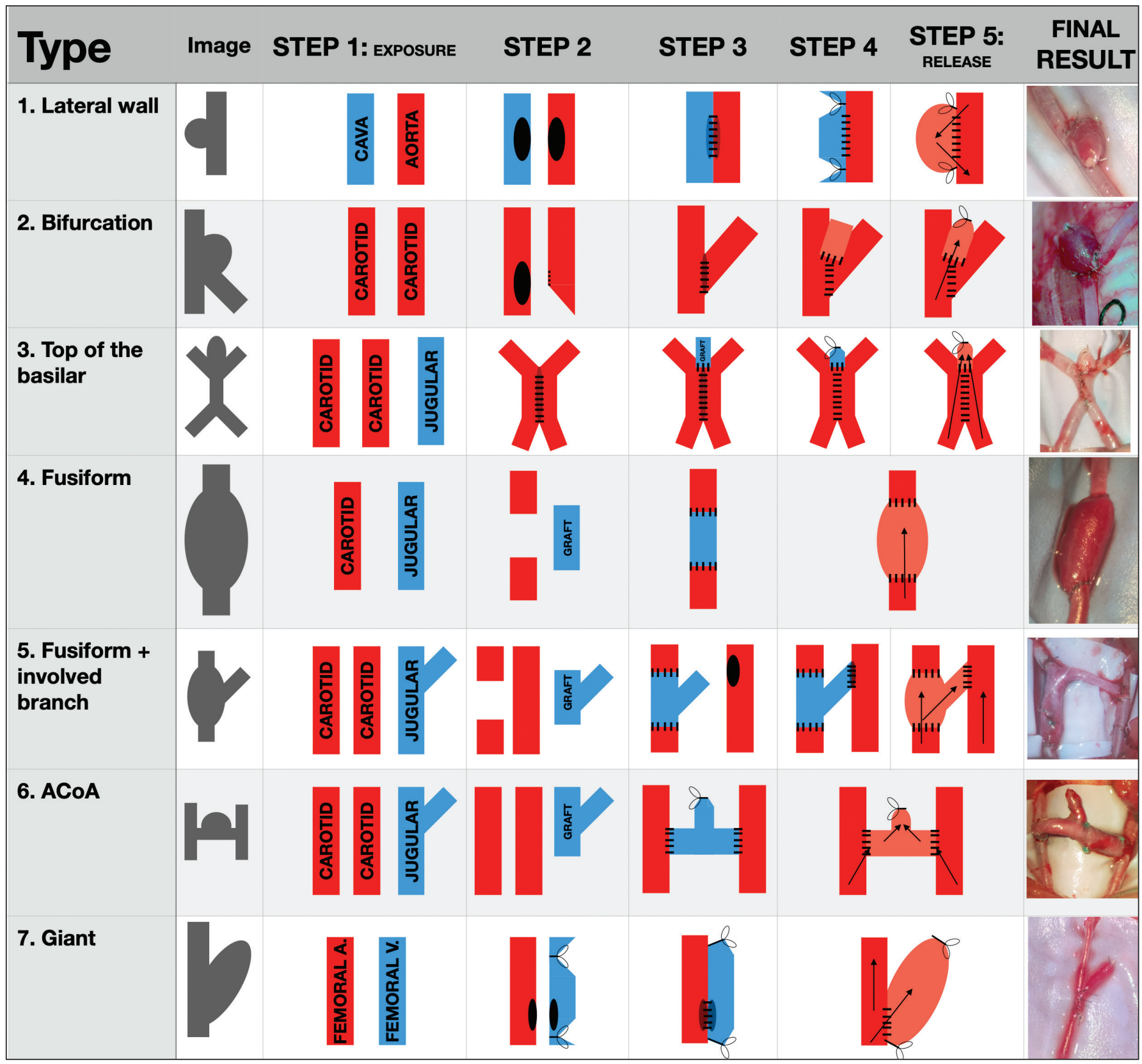

Figure 1: Schematic step-by-step guide, which includes a photo of the final result. (ACoA: Anterior communicating artery). 
2. Exposure of the carotid artery, temporal clipping and an excision arteriotomy on its lateral wall must be performed.

3. An end-to-side anastomosis between the carotid and the graft is made.

4. Ligation of the free edge of the graft, which would simulate the dome.

\section{Bifurcation:}

On the basis of previous works (9) and considering the number of involved vessels, we recommend practicing this exercise in the cervical segment.

1. Wide exposure of both carotids is performed with temporary clipping of one of them (recipient) above and below our surgical target. A temporary clip is placed on the distal part (above) of the donor artery with subsequent ligation of the proximal part (below) of the vessel in a permanent way.

2. An oblique transection arteriotomy in the donor carotid is made near the proximal clip and the surface of the lumen is enlarged in a "fish mouth" way.

3. An end-to-side anastomosis between both carotids is made without suturing a small portion in the upper part.

4. A small carotid or jugular graft is harvested and sutured over the hole to simulate an aneurysm located in the bifurcation. We should remember to keep enough carotid vessel during previous steps for grafting a small portion.

5. To complete the exercise, occlusion of the free edge of the graft with stitches must follow.

\section{Top of the basilar:}

This type is one of the most beautiful recreations in the cervical segment with three vessels involved.

1. After exposure and temporary clipping of both carotids, a side-to-side anastomosis is carried out.

2. It is necessary not suture the upper part, which will be the neck of the aneurysm later on.

3. A small jugular vein graft is harvested from the neck of the rat.

4. An end-to-side anastomosis between one edge of the jugular graft and the hole left in the upper surface of the arterial anastomosis is performed, preferably by using interrupted suture.

5. This exercise is completed by occluding the free edge of the jugular graft, which will simulate the dome of an aneurysm, arising from the top of the new created vessel (mimicking the basilar artery).

\section{Fusiform:}

Two vessels are involved in the recreation of this aneurysm: carotid and jugular.

1. Proximal and distal temporary ligation of the carotid artery.
2. A perpendicular transection arteriotomy is made in the middle of the vessel. A large jugular vein graft is collected from the neck of the rodent (collateral branches should be ligated).

3. Two end-to-end anastomoses are performed, with the venous graft placed between the two segments of carotid obtained with the previous arteriotomy.

4. An enlarged size of the venous graft compared to the arterial segments is produced when the blood flows through this segment.

\section{Fusiform plus:}

We designed this exercise by modifying the fusiform type steps.

1. Both carotids are exposed and temporarily clipped as it is previously described.

2. A jugular vein large graft that includes a branch (normally from the bifurcation) is collected. The rest of the collateral branches are ligated.

3. A similar procedure to the previous one (Fusiform aneurysm, step 3) is carried out with the vein branch placed medially, next to the contralateral carotid.

4. The distal end of the vein brach is anastomosed in an endto-side way to the other carotid, after the carotid temporary clipping and excision arteriotomy on its medial wall.

5. The result is a fusiform aneurysm that involves the normal circulation of both carotids.

\section{Anterior Communicating Artery (ACoA):}

This type of aneurysm requires a bypass between both carotids and it is an interesting training exercise for venous and arterial vessels manipulation.

1. At first both carotids and the jugular vein are exposed.

2. A huge venous graft from the jugular vein, which includes one branch in the middle, is obtained.

3. Two end-to-side anastomoses are performed between both carotids and the main lumen of the donor (jugular vein) perpendicularly. This way, a " $\mathrm{H}$ " structure is obtained with exchanging arterial flow between both carotid arteries through the vein graft. The jugular branch that was previously preserved must be upward or anteriorly oriented in this structure.

4. Final occlusion with stitches of the free edge of the jugular branch is performed. This branch is the masterpiece because it represents an aneurysm arising from the ACoA (jugular vein graft), located between the anterior cerebral arteries (carotids).

\section{Giant:}

This type is a conceptual modification from the saccular type. Low caliber vessels such as the femoral artery were selected to make an aneurysmal new vessel that mimics a huge one when it is compared under the microscope with 

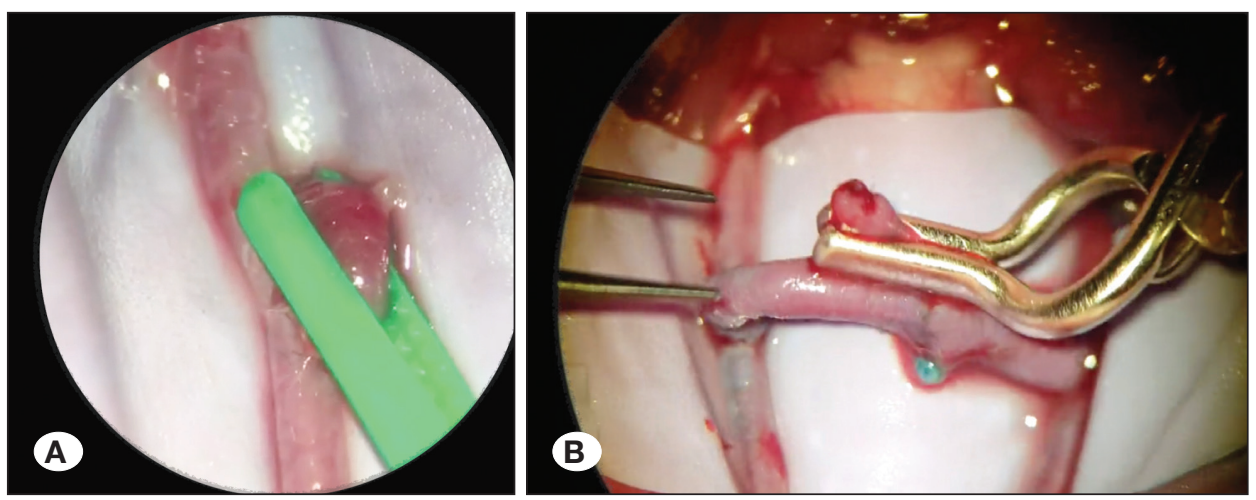

Figure 2: Aneurysm clipping:

A) Clipping of "lateral wall" aneurysm using a green vascular hemoclip.

B) Fenestrated curved mini-clip for an "ACoA" aneurysm.

(ACoA: Anterior Communicating Artery).

the surrounding vessels. This is one of the safest exercises we can practice on the animal, because some blood flow in the lower limb is preserved when the femoral artery is ligated (there is an extra-abdominal anastomotic circle between the internal and external iliac arteries) (1).

1. First at all the femoral vessels (artery and vein) are dissected and separated from each other.

2. Both vessels are ligated proximally and distally, but only the vein will be occluded permanently.

3. After that, the vessels are connected by using a side-toside anastomosis.

4. The femoral vein is ligated therefore its blood flow will be now provided by the artery through the anastomosis. With a section of the vein above and below the junction a vessel that mimics a giant aneurysm on the femoral artery wall is obtained. It is important to make the ligation of the vein far from the anastomosis to achieve a large size aneurysm.

\section{DISCUSSION}

Surgical education has a long history of using simulation training models to improve microsurgical skills. At present, high quality non-living models for vascular surgery are made using plastic, such as the PVC rat model (7), although they are suboptimal and not as similar as the rodent's (2,4-6,9-12). On the other hand, models such as chicken wing described by JOlabe and Olabe (9), are easily reproducible and obtained by the trainees without any implications about the life of the animal and offer good quality results in terms of aneurysm creation.

At the beginning of a training period it is recommended to work with non-living models (3), until basic microsurgery concepts and technical aptitudes are achieved. However, when we want to master the technique, we must develop microsurgical skills in more complex models with real blood flow through the vessels.

Whilst previous literature exists regarding aneurysm creation in rodent models (10), most just describe one or two models. For this reason, a broad range of vessel and anastomosis combinations have been included in this paper in order to offer challenging and different exercises differing from usual training models. Additionally, the good quality results shown by the aneurysms performance allowed for further clippings to be performed (Figure 2A, B).

The present paper highlights the value of the aneurysm design, without focusing on the description of basic experimental microvascular concepts (e.g., vein-graft harvesting, Wistar rat anatomy, or suturing technique tips). Our aim is to provide guidance to microsurgeons and even encourage some of them to develop and publish new exercises.

Despite the results obtained, the final size may vary from the regular real intracranial aneurysms, and we recommend choosing bigger live animal models for purposes other than those described.

Neurovascular surgeons are most likely to benefit from this proposed exercises, and even if they do not performed bypass surgery in their regular practice, they must be prepared in case the need should arise. We cannot forget that vascular techniques could be required at anytime, particularly in emergencies.

\section{CONCLUSION}

The feasibility for seven different types of aneurysm recreations has been successfully demonstrated in a rodent model, which can mimic those found in the human brain. The performance of these surgical manoeuvres will improve the skills and the confidence of the training surgeon.

\section{ACKNOWLEDGEMENTS}

I would like to thank SENEC (Sociedad Española de Neurocirugía) for awarding our e-poster entitled "Seven Aneurysms: experimental training model for aneurysm creation", which included portions of this work, for the 1st prize in the e-poster presentation of XXIII SENEC Congress in 2019 in Salamanca, which encouraged us to write this original paper.

\section{REFERENCES}

1. Amodeo CA: The need of a correct experimental model in microsurgery: An issue that may invalidate the resultsis. it time for an international scientific "Bank"? Microsurgery 37(5):469470, 2017 
García-Feijoo P. et al: Intracranial Aneurysms in Rodents

2. Evgeniou E, Walker H, Gujral S: The Role of simulation in microsurgical training. J Surg Educ 75(1):171-181, 2018

3. Frösen J, Tulamo R, Paetau A, Laaksamo E, Korja M, Laakso A, Niemelä M, Hernesniemi J: Saccular intracranial aneurysm: Pathology and mechanisms. Acta Neuropathol 123(6):773786, 2012

4. Hwang G, Wan Oh C, Park SQ, Sheen SH, Bang JS, Kang HS: Comparison of different microanastomosis training models: Model accuracy and practicality. J Korean Neurosurg Soc 47(4): 287-290, 2010

5. Lahiri A, Lim AYT, Qifen Z, Lim BH: Microsurgical skills training: A new concept for simulation of vessel-wall suturing. Microsurgery 25(1):21-24, 2005

6. Lawton MT: Preface. In: Lawton M, Probst K (eds), Seven Aneurysms: Tenets and Techniques for Clipping. Thieme, 2011: 6

7. Lawton MT: Preface. In: Lawton M, Probst K (eds), Seven Bypasses: Tenets and Techniques for Revascularization. Thieme, 2018:13

8. Marbacher S, Marjamaa J, Abdelhameed E, Hernesniemi J, Niemelä M, Frösen J: The Helsinki rat microsurgical sidewall aneurysm model. J Vis Exp 92:e51071, 2014
9. Olabe J, Olabe J: Microsurgical training on an in vitro chicken wing infusion model. Surg Neurol 72(6):695-699, 2009

10. Spetzger U, von Schilling A, Brombach T, Winkler G: Training models for vascular microneurosurgery. In: Tsukahara $T$, Regli L, Hänggi D, Turowski B, Steiger HJ (eds), Trends in Neurovascular Surgery. Acta Neurochirurgica Supplementum, vol: 112, Vienna: Springer, 2011:115-119

11. Stienen MN, Bartek J JR, Czabanka MA, Freyschlag CF, Kolias A, Krieg SM, Moojen W, Renovanz M, Sampron N, Adib SD, Schubert GA, Demetriades AK, Ringel F, Regli L, Schaller $\mathrm{K}$, Meling TR, for the EANS Young Neurosurgeons and EANS Training Committee: Neurosurgical procedures performed during residency in europe-preliminary numbers and time trends. Acta Neurochirurgica 161:843-853, 2019)

12. Tolba RH, Czigány $Z$, Lujan SO, Oltean $M$, Axelsson $M$, Akelina Y, Di Cataldo A, Miko I, Furka I, Dahmen U, Kobayashi $\mathrm{E}$, lonac $\mathrm{M}$, Nemeth N: Defining standards in experimental microsurgical training: Recommendations of the European Society for Surgical Research (ESSR) and the International Society for Experimental Microsurgery (ISEM). Eur Surg Res 58(5-6):246-262, 2017 